ORIGINAL ARTICLE

\title{
The study of functional asymmetry in students and schoolchildren practicing martial arts
}

Vyacheslav V. Romanenko ${ }^{1 \mathrm{ABCD}}$, Olha O. Podrihalo ${ }^{1 \mathrm{ABCD}}$, Leonid V. Podrigalo ${ }^{1,3 \mathrm{ABCD}}$, Sergii S. Iermakov ${ }^{2 \mathrm{ABCD}}$, Zhanna V. Sotnikova-Meleshkina ${ }^{3 \mathrm{ABCD}}$, Oksana V. Bobrova ${ }^{\mathrm{ABCD}}$

${ }^{1}$ Kharkiv State Academy of Physical Culture, Kharkov, Ukraine

${ }^{2}$ Gdansk University of Physical Education and Sport, Gdańsk, Poland

${ }^{3}$ V. N. Karazin Kharkov National University, Kharkov, Ukraine

Authors' Contribution: A - Study design; B - Data collection; C - Statistical analysis; D - Manuscript Preparation; E - Funds Collection.

\section{Abstract}

Purpose: $\quad$ The study of functional asymmetry in students and schoolchildren practicing martial arts using a computer test.

Material: $\quad$ The study involved students and schoolchildren $(n=38)$ practicing the martial arts (taekwondo, karate). Participants were divided into groups according to the sports' skill level. The first group included experienced athletes ( $n=15$, age $-19.00 \pm 0.45$ years). The second group included beginners ( $n=23$, age $-9.78 \pm 0.65$ years). The functional asymmetry was determined using «Reaction SM Dual» software for tablets with iOS. Two visual tests were used. The duration of each test was $100 \mathrm{~s}$. Each test was divided into 5 stages. Each stage lasts $20 \mathrm{~s}$. The first test (simple reaction) - the participant pushes against the circles on the screen with two hands. The second test (differentiated reaction) - the participant pushes against the circles of the same color (out of five possible) on the screen. The following indicators were determined: the number of pushes with the right and left hand (abs); reaction time with the right and left hand (s); duration of pushes with the right and left hand $(\mathrm{ms})$. It was estimated the following indicators: total number of pushes; average reaction time; average duration of pushes. Results are estimated using parametric and nonparametric statistical indicators.

Results: $\quad$ The number of pushes in the test, the reaction rate, and the duration of pushes were significantly higher in experienced athletes. It was determined the asymmetry due to the lower duration of pushes with the right hand compared to the left hand in beginners. The asymmetry was not confirmed in experienced athletes. The reaction rate for the left hand significantly increased compared to stage 1 in beginners: at stage $2(t=-2.41)$, at stage $3(t=-2.23)$, at stage $4(t=-2.30)$ and at stage $5(t=-2.68)$. This dependency was less expressed for the right hand. It was confirmed the increase of the reaction rate in comparison with stage 1 : at stage $3(t=-2.39)$, at stage $4(t=-2.00)$ and at stage $5(t=-2.32)$. The differences in the dynamics of the test were more expressed in experienced athletes. It was determined the decrease in the number of pushes compared to stage 1: stage $2(t=2.53)$, stage $4(t=2.89)$ and stage $5(t=2.61)$. For the right hand, this pattern was more expressed. The decrease in the number of pushes was determined compared with stage 1 : stage $2(t=2.17)$, stage $3(t=$ $2.07)$, stage $4(t=2.39)$ and stage $5(t=2.94)$. Experienced athletes have confirmed significant changes in the reaction rate with their left hand compared to stage 1 : stage $4(t=-2.33)$ and stage $5(t=-2.06)$. For the right hand, this pattern was confirmed only for the final stage of the test $(t=-2.35)$.

Conclusions: It was confirmed the legitimacy of using a special computer test to study functional asymmetry in students and schoolchildren practicing martial arts. The test division into several stages can significantly increase the information content of the results. The applied battery of indicators provides the necessary information for the analysis of asymmetry. It was determined the best functional condition of experienced athletes compared to beginners. Experienced athletes demonstrate the best ability to mobilize and concentrate on extreme conditions. The dynamics of the test also confirms the asymmetry in athletes with less training experience. Performing a test with a differentiated stimulus confirmed the revealed patterns. The lack of asymmetry in experienced athletes confirms optimal training tactics. The asymmetry in beginners stipulates the correction of training. Used computer test can be recommended for martial arts athletes' condition monitoring.

Keywords: functional asymmetry, martial arts, computer program, students, schoolchildren.

\section{Introduction}

The asymmetry of body functions is quite common. It is important in physical education and sports. According to many authors' opinion, this is a condition for effective motor action, including motor skills' training. Svetlana et al. [1] investigated the biological phenomenon of a person's functional asymmetry and its correlation with o Vyacheslav V. Romanenko, Olha O. Podrihalo, Leonid V. Podrigalo,

Sergii S. lermakov, Zhanna V. Sotnikova-Meleshkina,

Oksana V. Bobrova., 2020

doi:10.15561/20755279.2020.0305 sports results. It is concluded that it is necessary to consider the functional asymmetry in the training process. This allows providing the long-term positive dynamics of sports results.

The comprehensive analysis of a person's functional asymmetry was carried out by Khudik et al. [2]. The main types of functional asymmetry are motor, sensory, and psychic. In the course of the review of research results in some sports, the effect of the functional asymmetry on the athletic performance was assessed. There are works 
where the same sport is pointed out as having a positive influence of asymmetry by some authors, or as having a negative effect by others. It can be concluded that functional asymmetry is a biological phenomenon due to which it is possible to avoid an early formation of motor skills.

The asymmetry studies are often used in sports. Hernandez-Garcia et al. [3] emphasize that asymmetry analysis is a simple, economical and informative tool for the athletes' condition monitoring.

Caraballo et al. [4] studied the asymmetry of morphofunctional indicators in yachtsmen with different skills' levels. It is proposed to use asymmetry indicators as predictors of success.

Teixeira et al. [5] used the study of functional asymmetry in assessing the quality of high-intensity functional training. Gomez Piqueras et al. [6] developed benchmarks to assess the skills' level of professional football players. The asymmetry indicators are included in the list of proposed criteria.

Arede et al. [7] determined the selection criteria of young basketball players. The success predictors include asymmetry indicators. Tabor et al. [8] studied the correlation between the level of functional and dynamic asymmetry and running speed in elite and sub-elite runners. It is concluded that a decrease in asymmetry leads to an increase in running speed results.

Warmenhoven et al. [9] investigated the asymmetry in elite rowers by assessing the symmetry index and functional data analysis. Differences in symmetry are a competition level factor. Elite rowers use the asymmetry of movements to increase the strength and speed of movements.

Thus, the available literature data confirm the relevance of the study of functional asymmetry in athletes practicing the different types of sports.

The purpose of this work was to study functional asymmetry in students and schoolchildren practicing martial arts using a computer test.

\section{Materials and methods}

Participants.

The study involved students and schoolchildren ( $\mathrm{n}=38$ ) practicing martial arts (taekwondo, karate). Participants were divided into groups according to the sports' skill level. The first group included experienced athletes $(\mathrm{n}=15$, age $-19.00 \pm 0.45$ years $)$. The second group included beginners $(\mathrm{n}=23$, age $-9.78 \pm 0.65$ years $)$.

\section{Research Design}

The study design involved the determination of functional asymmetry using «Reaction SM Dual» software. The program is designed for tablets with iOS [10]. Two visual tests were used to assess the functional asymmetry of athletes. Tests are differed in the complexity of the visual stimuli influence on the performer. The first test (simple reaction): the participant must push with the same hands against the same visual signals (blue circles). In performing the second test (differentiated reaction), the participant must push only against the blue circles. The feature of this task is the stimulus: circles of other colors also appear on the screen with blue circles. Each test was divided into 5 stages. Each stage lasts $20 \mathrm{~s}$. The duration of each test is $100 \mathrm{~s}$.

The following indicators were determined: the number of pushes with the right and left hand (abs); reaction time with the right and left hand (s); duration of pushes with the right and left hand (ms). It was estimated: total number of pushes; average reaction time; average duration of pushes.

It was compared the results of tests in groups, the dynamics of tests, and the results of groups.

Statistical analysis.

Statistical analysis of the obtained data was carried out using licensed MS Excel. It was determined the following indicators of descriptive statistics: arithmetic mean, standard deviation, and mean error. The significance of differences in the groups was estimated using parametric (Student's test) and nonparametric (Rosenbaum's test) indicators. Differences were considered significant at $\mathrm{p}$ $<0.05$.

\section{Results}

The results reflect the simple reaction performance (table 1) and a differentiated reaction (table 2).

The results of table 1 illustrate the significant differences between the groups for all studied indicators. The number of pushes with the left hand was significantly higher in the group of experienced athletes at all stages (respectively, from stage 1 to 5): $(\mathrm{t}=4.87),(\mathrm{t}=6.82),(\mathrm{t}$ $=6.06),(\mathrm{t}=6.17),(\mathrm{t}=7.05)$. The total number of pushes with the left hand was also differed significantly $(\mathrm{t}=$ 21.75). Experienced athletes were characterized by the best reaction time with their left hand in performing the test at all stages (stages 1 to 5, respectively): $(\mathrm{t}=52.38),(\mathrm{t}$ $=52.29),(\mathrm{t}=52.29),(\mathrm{t}=52.28),(\mathrm{t}=52.28)$. Experienced athletes had the best average reaction time $(\mathrm{t}=52.30)$. The improvement in reaction time led to a reduction in the duration of push in all stages in experienced athletes (respectively from stage 1 to 5$)$ : $(\mathrm{t}=10.02),(\mathrm{t}=9.65),(\mathrm{t}$ $=7.91, \mathrm{Q}=7),(\mathrm{t}=8.65, \mathrm{Q}=10),(\mathrm{t}=9.18, \mathrm{Q}=9)$ and the average value of the duration of the push $(\mathrm{t}=10.28)$.

The similar correlation is confirmed for the right hand. The number of pushes was higher in experienced athletes in all stages (stages 1 to 5 , respectively): $(\mathrm{t}=4.33),(\mathrm{t}=$ $5.11),(\mathrm{t}=6.12),(\mathrm{t}=5.44),(\mathrm{t}=6.45)$, and by the total number of pushes $(t=22.27)$. Experienced athletes had the best reaction rate indicators at all stages of the test (stage 1 to 5, respectively): $(\mathrm{t}=52.39, \mathrm{Q}=7),(\mathrm{t}=52.31)$, $(\mathrm{t}=52.30),(\mathrm{t}=52.31),(\mathrm{t}=52.31)$. This pattern is also confirmed for the average reaction time $(\mathrm{t}=52.33)$. The duration of pushes with the right hand in experienced athletes was also significantly lower. This is confirmed by both the Student criterion and the Rosenbaum criterion. Their values were for the test stages (respectively, from stage 1 to 5$):(t=8.04),(t=7.80),(t=6.18),(t=7.24, Q$ $=8),(\mathrm{t}=7.76, \mathrm{Q}=9)$, for the average duration of pushes $(\mathrm{t}=8.63, \mathrm{Q}=7)$.

Beginners were characterized by the asymmetry in performing a simple test. This resulted in a lower duration 
Table 1. Simple reaction performance for functional asymmetry by athletes with different skills' levels.

\begin{tabular}{|c|c|c|}
\hline Indicator & Experienced athletes $(n=15)$ & Beginners $(n=23)$ \\
\hline The number of pushes with the left hand - 1 stage, abs & $41.93 \pm 0.64^{1}$ & $35.30 \pm 1.11$ \\
\hline The number of pushes with the left hand -2 stage, abs & $39.67 \pm 0.62^{1}$ & $32.65 \pm 1.11$ \\
\hline The number of pushes with the left hand -3 stage, abs & $40.40 \pm 0.56^{1}$ & $32.87 \pm 1.27$ \\
\hline The number of pushes with the left hand -4 stage, abs & $39.33 \pm 0.63^{1}$ & $32.52 \pm 1.31$ \\
\hline The number of pushes with the left hand -5 stage, abs & $39.40 \pm 0.73^{1}$ & $32.22 \pm 1.13$ \\
\hline The total number of pushes with the left hand, abs & $200.73 \pm 2.71^{1}$ & $165.57 \pm 5.63$ \\
\hline Left hand reaction time -1 stage, $s$ & $0.48 \pm 0.01^{1}$ & $0.57 \pm 0.02$ \\
\hline Left hand reaction time -2 stage, $\mathrm{s}$ & $0.50 \pm 0.01^{1}$ & $0.63 \pm 0.02$ \\
\hline Left hand reaction time -3 stage, $s$ & $0.50 \pm 0.01^{1}$ & $0.63 \pm 0.02$ \\
\hline Left hand reaction time -4 stage, $\mathrm{s}$ & $0.51 \pm 0.01^{1}$ & $0.63 \pm 0.03$ \\
\hline Left hand reaction time -5 stage, $\mathrm{s}$ & $0.51 \pm 0.01^{1}$ & $0.64 \pm 0.02$ \\
\hline The average reaction time with the left hand, $s$ & $0.50 \pm 0.01^{1}$ & $0.62 \pm 0.02$ \\
\hline Duration of push with the left hand - 1 stage, ms & $55.15 \pm 3.27^{1}$ & $70.20 \pm 2.71^{2}$ \\
\hline Duration of push with the left hand -2 stage, $\mathrm{ms}$ & $54.79 \pm 3.41^{1}$ & $73.10 \pm 3.13^{2}$ \\
\hline Duration of push with the left hand -3 stage, ms & $56.11 \pm 3.60^{1}$ & $73.76 \pm 3.94^{2}$ \\
\hline Duration of push with the left hand -4 stage, $\mathrm{ms}$ & $56.41 \pm 4.05^{1}$ & $73.76 \pm 3.80^{2}$ \\
\hline Duration of push with the left hand -5 stage, $\mathrm{ms}$ & $55.01 \pm 4.10^{1}$ & $77.34 \pm 3.77^{2}$ \\
\hline The average duration of push with the left hand, ms & $55.49 \pm 3.57^{1}$ & $73.97 \pm 3.02^{2}$ \\
\hline The number of pushes with the right hand - 1 stage, abs & $42.40 \pm 0.65^{1}$ & $36.04 \pm 1.11$ \\
\hline The number of pushes with the right hand -2 stage, abs & $40.33 \pm 0.69^{1}$ & $34.00 \pm 1.34$ \\
\hline The number of pushes with the right hand -3 stage, abs & $40.33 \pm 0.58^{1}$ & $33.09 \pm 1.21$ \\
\hline The number of pushes with the right hand -4 stage, abs & $40.13 \pm 0.69^{1}$ & $33.65 \pm 1.30$ \\
\hline The number of pushes with the right hand -5 stage, abs & $39.73 \pm 0.63^{1}$ & $33.26 \pm 1.09$ \\
\hline Total number of pushes with the right hand, abs & $203.20 \pm 2.91^{1}$ & $170.04 \pm 5.70$ \\
\hline Right hand reaction time -1 stage, $s$ & $0.48 \pm 0.01^{1}$ & $0.56 \pm 0.01$ \\
\hline Right hand reaction time -2 stage, $\mathrm{s}$ & $0.48 \pm 0.01^{1}$ & $0.61 \pm 0.02$ \\
\hline Right hand reaction time -3 stage, $s$ & $0.50 \pm 0.01^{1}$ & $0.62 \pm 0.02$ \\
\hline Right hand reaction time -4 stage, $\mathrm{s}$ & $0.50 \pm 0.01^{1}$ & $0.61 \pm 0.02$ \\
\hline Right hand reaction time -5 stage, $\mathrm{s}$ & $0.50 \pm 0.01^{1}$ & $0.61 \pm 0.02$ \\
\hline The average reaction time with the right hand, $\mathrm{s}$ & $0.49 \pm 0.01^{1}$ & $0.60 \pm 0.02$ \\
\hline Duration of push with the right hand - 1 stage, ms & $53.64 \pm 2.74^{1}$ & $60.92 \pm 2.23$ \\
\hline Duration of push with the right hand - 2 stage, $\mathrm{ms}$ & $52.47 \pm 2.83^{1}$ & $60.04 \pm 2.18$ \\
\hline Duration of push with the right hand - 3 stage, ms & $51.31 \pm 3.13^{1}$ & $60.73 \pm 2.94$ \\
\hline Duration of push with the right hand -4 stage, $\mathrm{ms}$ & $50.83 \pm 3.04^{1}$ & $62.73 \pm 2.76$ \\
\hline Duration of push with the right hand -5 stage, $\mathrm{ms}$ & $49.91 \pm 3.09^{1}$ & $60.90 \pm 2.31$ \\
\hline Average duration of push with the right hand, ms & $51.64 \pm 2.84^{1}$ & $61.06 \pm 2.07$ \\
\hline
\end{tabular}

NOTE: 1 - differences with beginners are significant, 2 - differences with the right hand are significant.

of pushes with the right hand compared to the left hand. Such a dependency was determined at all stages of the test (stage 1 to 5 , respectively): $(\mathrm{t}=2.65),(\mathrm{t}=3.42),(\mathrm{t}=$ $2.65),(\mathrm{t}=2.72),(\mathrm{t}=3.71)$ and the average value of this indicator $(\mathrm{t}=3.53)$.

A comparison of the results in the dynamics of the test also found some differences. The response time for the left hand significantly increased compared to stage 1 in beginners: at stage $2(\mathrm{t}=-2.41)$, at stage $3(\mathrm{t}=-2.23)$, at stage $4(\mathrm{t}=-2.30)$ and at stage $5(\mathrm{t}=-2.68)$. For the right hand, this dependency was less expressed. The increase in the reaction time in comparison with the $1^{\text {st }}$ stage was confirmed: at the $3^{\text {rd }}$ stage of the test $(\mathrm{t}=-2.39)$, at the $4^{\text {th }}$ stage $(\mathrm{t}=-2.00)$, and the $5^{\text {th }}$ stage $(\mathrm{t}=-2.32)$.
The differences in the dynamics of the test were more expressed in experienced athletes. The decrease in the number of pushes was determined in comparison with the $1^{\text {st }}$ stage of the test: at the $2^{\text {nd }}$ stage $(t=2.53)$, the $4^{\text {th }}$ stage $(\mathrm{t}=2.89)$, and the $5^{\text {th }}$ stage $(\mathrm{t}=2.61)$. This pattern was more expressed for the right hand. The reduction in the number of pushes was determined in comparison with the first stage of the test: at the second stage $(\mathrm{t}=2.17)$, the third stage $(\mathrm{t}=2.07)$, the fourth stage $(\mathrm{t}=2.39)$, and the fifth stage $(t=2.94)$. The significant changes in the reaction rate with left hand compared to stage 1 were confirmed in experienced athletes: stage $4(\mathrm{t}=-2.33)$ and stage $5(t=-2.06)$. This pattern was confirmed only for the final stage of the test for the right hand $(t=-2.35)$. 
Table 2. The differentiated test performance of functional asymmetry by athletes with different skills' levels

\begin{tabular}{|c|c|c|}
\hline Indicator & Experienced athletes $(n=15)$ & Beginners $(n=23)$ \\
\hline The number of pushes with the left hand - 1 stage, abs & $34.93 \pm 0.79^{1}$ & $29.59 \pm 1.24$ \\
\hline The number of pushes with the left hand -2 stage, abs & $33.73 \pm 0.64^{1}$ & $26.59 \pm 1.36$ \\
\hline The number of pushes with the left hand -3 stage, abs & $34.07 \pm 0.52^{1}$ & $27.41 \pm 1.34$ \\
\hline The number of pushes with the left hand -4 stage, abs & $33.73 \pm 0.61^{1}$ & $27.50 \pm 1.42$ \\
\hline The number of pushes with the left hand -5 stage, abs & $34.13 \pm 0.56^{1}$ & $27.55 \pm 1.33$ \\
\hline The total number of pushes with the left hand, abs & $170.60 \pm 2.67^{1}$ & $138.64 \pm 6.55$ \\
\hline Left hand reaction time -1 stage, $s$ & $0.58 \pm 0.01^{1}$ & $0.71 \pm 0.03$ \\
\hline Left hand reaction time -2 stage, $\mathrm{s}$ & $0.58 \pm 0.01^{1}$ & $0.79 \pm 0.04$ \\
\hline Left hand reaction time -3 stage, $\mathrm{s}$ & $0.59 \pm 0.01^{1}$ & $0.77 \pm 0.04$ \\
\hline Left hand reaction time -4 stage, $\mathrm{s}$ & $0.59 \pm 0.01^{1}$ & $0.77 \pm 0.04$ \\
\hline Left hand reaction time -5 stage, $s$ & $0.59 \pm 0.01^{1}$ & $0.76 \pm 0.04$ \\
\hline The total number of pushes with the left hand, $s$ & $0.59 \pm 0.01^{1}$ & $0.76 \pm 0.04$ \\
\hline Duration of push with the left hand - 1 stage, ms & $59.49 \pm 3.70^{1}$ & $72.18 \pm 2.69^{2}$ \\
\hline Duration of push with the left hand -2 stage, $\mathrm{ms}$ & $58.69 \pm 4.86^{1}$ & $75.64 \pm 2.92^{2}$ \\
\hline Duration of push with the left hand -3 stage, ms & $59.66 \pm 4.55^{1}$ & $74.48 \pm 3.39^{2}$ \\
\hline Duration of push with the left hand -4 stage, ms & $56.61 \pm 4.14^{1}$ & $74.75 \pm 3.39^{2}$ \\
\hline Duration of push with the left hand -5 stage, $\mathrm{ms}$ & $57.20 \pm 4.08^{1}$ & $74.23 \pm 3.60^{2}$ \\
\hline The average duration of push with the left hand, ms & $58.32 \pm 4.17^{1}$ & $74.25 \pm 3.00^{2}$ \\
\hline The number of pushes with the right hand -1 stage, abs & $35.27 \pm 0.75^{1}$ & $30.32 \pm 1.34$ \\
\hline The number of pushes with the right hand -2 stage, abs & $34.20 \pm 0.64^{1}$ & $27.36 \pm 1.37$ \\
\hline The number of pushes with the right hand -3 stage, abs & $34.73 \pm 0.61^{1}$ & $28.18 \pm 1.42$ \\
\hline The number of pushes with the right hand -4 stage, abs & $34.47 \pm 0.84^{1}$ & $27.91 \pm 1.38$ \\
\hline The number of pushes with the right hand -5 stage, abs & $34.60 \pm 0.85^{1}$ & $28.00 \pm 1.34$ \\
\hline Total number of pushes with the right hand, abs & $173.27 \pm 3.26^{1}$ & $141.77 \pm 6.70$ \\
\hline Right hand reaction time -1 stage, $\mathrm{s}$ & $0.58 \pm 0.01^{1}$ & $0.70 \pm 0.03$ \\
\hline Right hand reaction time -2 stage, $\mathrm{s}$ & $0.59 \pm 0.01^{1}$ & $0.77 \pm 0.04$ \\
\hline Right hand reaction time -3 stage, $\mathrm{s}$ & $0.58 \pm 0.01^{1}$ & $0.75 \pm 0.04$ \\
\hline Right hand reaction time -4 stage, $\mathrm{s}$ & $0.58 \pm 0.01^{1}$ & $0.75 \pm 0.04$ \\
\hline Right hand reaction time -5 stage, $\mathrm{s}$ & $0.58 \pm 0.01^{1}$ & $0.74 \pm 0.04$ \\
\hline The average reaction time with the right hand, $s$ & $0.58 \pm 0.01^{1}$ & $0.74 \pm 0.04$ \\
\hline Duration of push with the right hand - 1 stage, ms & $53.73 \pm 2.96^{1}$ & $61.62 \pm 2.48$ \\
\hline Duration of push with the right hand -2 stage, $\mathrm{ms}$ & $54.07 \pm 3.08^{1}$ & $63.28 \pm 2.41$ \\
\hline Duration of push with the right hand -3 stage, ms & $53.44 \pm 3.04^{1}$ & $61.92 \pm 2.81$ \\
\hline Duration of push with the right hand -4 stage, ms & $51.70 \pm 3.02^{1}$ & $64.00 \pm 3.01$ \\
\hline Duration of push with the right hand -5 stage, $\mathrm{ms}$ & $49.85 \pm 2.92^{1}$ & $62.94 \pm 3.14$ \\
\hline Average duration of push with the right hand, ms & $52.56 \pm 2.89^{1}$ & $62.76 \pm 2.60$ \\
\hline
\end{tabular}

NOTE: 1 - differences with beginners are significant, 2 - differences with the right hand are significant.

The increasing complexity of the test conditions due to the introduction of differentiated stimuli led to a decrease in indicators compared with the results of a simple test. However, the previously determined patterns are reserved. Experienced athletes had a significantly higher number of pushes compared to beginners at all stages of performing this test with the left hand (stages 1 to 5 , respectively): $(\mathrm{t}=$ 4.19, $\mathrm{Q}=13),(\mathrm{t}=6.55, \mathrm{Q}=17),(\mathrm{t}=5.91),(\mathrm{t}=5.83, \mathrm{Q}=$ $18),(t=5.80)$ and the total number of pushes $(t=81.39)$. The reaction rate with the left hand was also significantly higher at all stages (respectively from stage 1 to 5$)$ : $(\mathrm{t}=$ -26.86), $(\mathrm{t}=-26.79, \mathrm{Q}=14),(\mathrm{t}=-26.81),(\mathrm{t}=-26.81),(\mathrm{t}=$ $-26.82)$ and according to the average indicator $(t=-26.82)$.
As in a simple test, the duration of push in experienced athletes was significantly lower at all stages of the test (stages 1 to 5, respectively): $(\mathrm{t}=29.23),(\mathrm{t}=31.94, \mathrm{Q}=7)$, $(\mathrm{t}=31.04),(\mathrm{t}=31.25, \mathrm{Q}=9),(\mathrm{t}=30.84)$ and according to the average indicator $(\mathrm{t}=30.86, \mathrm{Q}=8)$.

A similar dependency was determined according to the results of the test with the right hand. The results of athletes of both groups were lower than in a simple test performing. Experienced athletes in all indicators had better results than beginners. They performed more pushes with left hand at all stages (respectively from stages 1 to 5$)$ : $(\mathrm{t}=-3.62),(\mathrm{t}=-5.94, \mathrm{Q}=16),(\mathrm{t}=-5.30)$, $(\mathrm{t}=-5.51, \mathrm{Q}=18),(\mathrm{t}=-5.44, \mathrm{Q}=14)$, had a significantly 
higher number of pushes per test $(t=83.85, Q=14)$. The reaction rate with the right hand was better at all stages of the test (respectively from stage 1 to 5$)$ : $(\mathrm{t}=-26.87),(\mathrm{t}=$ -26.81, $\mathrm{Q}=14), 3(\mathrm{t}=-26.83),(\mathrm{t}=-26.83, \mathrm{Q}=17),(\mathrm{t}=$ $-26.83, Q=12)$, and according to the average indicator $(\mathrm{t}$ $=-26.83, \mathrm{Q}=14)$. The duration of pushes with the right hand was also significantly lower at all stages of the test (respectively from stages 1 to 5$)$ : $(\mathrm{t}=20.95),(\mathrm{t}=22.25)$, $(\mathrm{t}=21.18, \mathrm{Q}=7),(\mathrm{t}=22.81),(\mathrm{t}=21.98, \mathrm{Q}=8)$ and according to the average indicator $(\mathrm{t}=21.84)$.

It was determined the expressed asymmetry of indicators in beginners. It is also true in a simple reaction test performing. The results of the duration of pushes were significantly higher with the left hand at all stages of the test (respectively from stage 1 to 5$):(t=2.89),(t=$ 3.27, $\mathrm{Q}=11),(\mathrm{t}=2.86, \mathrm{Q}=8),(\mathrm{t}=2.37),(\mathrm{t}=2.37)$ and according to the average indicator $(t=2.89, \mathrm{Q}=7)$. The complication of the task did not lead to the asymmetry in experienced athletes.

No significant differences were determined in the dynamics of the test with a differentiated stimulus.

\section{Discussion}

Visual tracking of a moving target is a sensorimotor function that depends on attention. Many sports require athletes to quickly transform visual information into a targeted motor reaction (visual-motor reaction). Understanding the neural functions that determine working capacity allows improving the athletes' diagnosis; increase productivity by improving training.

Psychophysiological characteristics of athletes reflect the specificity of the influence of the type of martial arts. Podrigalo et al. [11] determined that different types of martial arts are characterized by different predictors of success. The development and optimization of the selected qualities can improve the growth prospects of sports skills.

The importance of functional asymmetry for success in martial arts was confirmed by Nikolaenko et al. [12]. Data about peculiarities of organization of motor control and brain functional asymmetry pattern in highly trained judo wrestlers are presented. It has been shown that in athletes the right hemisphere is predominant in the processing of both speech and visual-spatial information, while high competition results in wrestlers with left-side stand correlate with the prevalence of the left hemisphere in speech perception. It is suggested that high sports results in wrestlers are due to the formation of novel long-term skills, reorganization of motor control, and interhemispheric interaction.

The used design involved a comparison of athletes practicing the same sport, differing in the sports' skill level. This allows evaluating the importance of asymmetry to achieve a high skill level.

A similar design was used by Rovnaya et al. [13]. The comparison of the functional condition of synchronized swimming athletes of different skill levels made it possible to distinguish the qualities related to success predictors.

Krstulovic et al. [14] determined the differences between elite and sub-elite female judo athletes by the level of morphological, functional, and dynamic asymmetry. The criterion for dividing into groups was the level of competitive success. The highest asymmetry of the hands' development was determined using the Edinburgh Handedness Inventory (EHI).

The study of functional asymmetry requires the use of various and functional tests. The functional movement screen (FMS) is used to determine the asymmetry. This is a battery of functional tests simulating various movements. Lewis et al. [15] indicate that it is an affordable and noninvasive tool for identifying weakness and asymmetry during specific exercises. FMS is used as a screening method for athletes in many sports. So Zhu J. [16] used it to study the causes of sports injuries and creating a model for predicting sports injuries in badminton.

Marques et al. [17] used the FMS test battery in young football players from Top League. It was confirmed the presence of functional disorders and a high prevalence of asymmetry between the right and left parts of the body.

de Oliveira et al. [18] used FMS to compare the biomechanical characteristics of runners. The special asymmetry index (IS) was used for analysis. The indicator was calculated as the ratio of the average score of a nondominant hand to the average score of a dominant hand, multiplied by 100 .

A review by Whittaker et al. [19] is indicated that the Functional Movement Screen, a battery of 7 motor tests, is most often used to assess functional asymmetry. This does not allow providing a qualitative morphofunctional assessment of the athletes' condition. The authors point to the need for using other tools for functional asymmetry analyzing.

The test we used contained two options (models). It allows reducing the testing time, the level of errors due to the influence of test loads on the athlete.

Wieczorek [20] used the following indicators to evaluate asymmetry: 8 tests determining the dominance of the eyes and hands; 5 tests evaluating the static balance, strength, accuracy, and speed of hands' movement, muscle strength of the fingers flexors. Studies have shown that the speed of learning a complex motor skill substantially depends on the determined direction and profile of functional asymmetry and motor skills.

Another test used to study asymmetry is tensiomyography. In a review by Garcia-Garcia et al. [21] emphasizes its significance for elite athletes' condition analyzing. The authors point out the lack of clear criteria for asymmetry. Arboix-Alio et al. [22] evaluated the effectiveness of various neuromuscular tests for assessing asymmetry in roller hockey. A large number of tests increases the significance of the results. Burmistrov et al. [23] used video analysis of movements and determination of many biomechanical indicators for the analysis of functional asymmetry in speed skating. Krzykala et al. [24] suggested using a goniometric study to analyze the asymmetry in field hockey players. The athletes confirmed the expressed asymmetry of morphological and functional indicators. The results are evaluated as a 
factor of the musculoskeletal system injuries.

The choice of a special computer test for asymmetry analysis is quite widespread in sports. Akinina et al. [25] used the Sports Psychophysiologist computer program to analyze the psychomotor development indicators of children who practiced karate. The effectiveness of using a computer program to assess the quality of training in martial arts has been confirmed. Hromcik et al. [26] used a similar research design to assess the speed of the sensorimotor reaction in badminton athletes. Participants performed a special computer test. The reaction rate was tested in various tasks with changing conditions for representing the target.

The computer program "Reaction SM Dual" has several advantages that allow recommending it for use [10]. These include efficiency, mobility, painlessness, and visibility of the results. Dividing the test into several stages can significantly increase the information content of the results. A similar technique was used by Podrigalo et al. [27] in the analysis of the psychophysiological condition of water and game sports athletes. Repetition of the same tests by participants allows indirectly to judge their ability to mobilize and concentrate in extreme conditions. The duration of the used tests is minimal and does not cause fatigue. Differences in the results allow assessing the stability and balance of the nervous system of the participants.

Identification of asymmetry is most optimal when performing any type of activity simultaneously with both hands. A similar design was used by Rodrigues et al. [28]. Participants performed the coincidence-anticipation task test both separately with right and left hands, and with both hands simultaneously. The results improved the asymmetry analysis.

The best results of a simple and differentiated test performing by experienced athletes reflect the best level of their functional condition. Experienced athletes complete the task faster. Therefore, they increase the number of pushes, improves the reaction time to the stimulus. The best mobilization rate is reflected in the reduction of the time for pushing the screen of the device. That is, experienced athletes win according to all test indicators.

Similar results are reported by Surina-Marysheva et al. [29]. The authors studied the features of sensorimotor integration and coordination of movements in elite hockey players using NS-PsychoTest software and hardware. Compared with peers, hockey players aged 15-16 years have a higher level of functional indicators in conditions of interference ( $p<0.05$ in all cases) and a better indicator of the excitation concentration. It is concluded that the specific conditions of the competitive activity of hockey players increase the efficiency of the central nervous system. This occurs due to an increase in the excitation concentration in conditions of interference. The sensorimotor integration of players of this age is also characterized by better predictive accuracy in reactions to a moving object.

The determined asymmetry of indicators in athletes' beginners confirms the adequacy and information content of the used test. These results allow judging the level of athletes' skills. In the process of martial arts athletes' training, they are trained to act equally with both hands, throw punch both the right and left hands. Experienced trainers pay attention to the asymmetry, trying to minimize it as much as possible. This gives athletes an additional advantage since throw punch from two sides significantly increases the probability of victory. In this context, the lack of asymmetry should be assessed as a success factor. In turn, the asymmetry in beginners is proof of an insufficient level of training and requires the training process correction.

An analysis of the test performing dynamics for a simple reaction In beginners suggests the difficulty of constantly maintaining the required functional level. This is evidenced by slowing down the reaction rate; decrease in the number of pushes; a sufficiently large number of stages at which these changes are confirmed. Such factors are less expressed on the right hand, which is the lead. An analysis of the results in the dynamics of the test also confirms the functional asymmetry in athletes with less training experience. Experienced athletes had differences in the manifestation of this pattern. Changes in the reaction rate were expressed only at the final stages of the test.

A decrease in the test performance of the with a differentiated stimulus was determined compared with a simple test. This illustrates the complexity of the task that participants have to complete. In this case, the decisionmaking process is complicated by the need to choose the right reaction object. In a simple reaction, the response algorithm consists of two stages: the appearance of the object - the reaction to it. In the second case, it lengthens the appearance of an object - its recognition - reaction. Naturally, this leads to significant changes in all studied indicators.

The increase in stress during the performance of a complicated test led to more expressed manifestations of already determined patterns. In this context, conducting two test variants allows us to assess the tendency to change, which is not manifested in the case of a simple reaction test.

For athletes with less training experience, the complexity of the task increases the asymmetry. This is confirmed by the results of the duration of pushes at all stages of the test. For experienced athletes, the complication of the task did not lead to the asymmetry. This can be evaluated as a significant level of significance, sufficient potential for controlling neuromuscular mechanisms.

\section{Conclusions}

The obtained results confirm the legitimacy of using a special computer test to study functional asymmetry in students and schoolchildren practicing martial arts. Dividing the test into several stages can significantly increase the information content of the results. The applied set of indicators provides the necessary information for the asymmetry analysis. It was defined as the best functional condition of experienced martial 
arts athletes in comparison with beginners has been determined. Experienced athletes demonstrate the best ability to mobilize and concentrate on extreme conditions. The dynamics of the test also confirms the asymmetry of athletes with short training experience. Performing a test with a differentiated stimulus confirmed the patterns identified. The lack of asymmetry in experienced athletes confirms optimal training tactics. The presence of asymmetry in beginners necessitates the correction of training. Used computer tests can be recommended for monitoring the condition of martial arts athletes.

\section{Conflicts of Interest}

The authors declare no conflict of interest.

\section{References}

1. Svetlana K, Aleksandr C, Marina P, Andrey B. Functional asymmetry and the sports result. Journal of Physical Education and Sport. 2018;18: 408-415. https://doi.org/10.7752/jpes.2018.s157

2. Khudik SS, Chikurov AI, Voynich AL, Radaeva SV. Functional asymmetry as a biological phenomenon associated with athletic performance. Tomsk State University Journal, 2017; (421): 193-202. https://doi.org/10.17223/15617793/421/29

3. Hernández-García R, Aparicio-Sarmiento A, Palao JM, Sainz de Baranda P. Influencia de las lesiones previas en los patrones fundamentales del movimiento en jugadoras profesionales de fútbol. [Influence of previous injuries on fundamental movement patterns in professional female soccer players]. Rev Int Cienc Deporte, 2020;16:214-35. (In Spanish) https://doi.org/10.5232/ricyde2020.06007

4. Caraballo I, Luis Gonzalez-Montesinos J, Alias A. Bilateral and Unilateral Asymmetries of Strength and Flexibility in Young Elite Sailors: Windsurfing, Optimist and Laser Classes. Symmetry-Basel. 2020; 12(1): e184. https://doi.org/10.3390/sym12010184

5. Teixeira RV, de Queiros VS, Dantas MP, Assis MG, Dantas PMS, de Araújo Tinôco Cabral BG. Asymmetry inter-limb and performance in amateur athletes involved in high intensity functional training. Isokinetics and Exercise Science. 2020; 28(1): 83-89. https://doi.org/10.3233/IES-194201

6. Gomez-Piqueras P, Gonzalez-Villora S, SanchezGonzalez M, Sainz de Baranda P. The functional assessment as a key element in the recovery of football players after an injury. Sportk, 2020:15-25. https://doi.org/10.6018/sportk.412461

7. Arede J, Ferreira AP, Gonzalo-Skok O, Leite N. Maturational Development as a Key Aspect in Physiological Performance and National-Team Selection in Elite Male Basketball Players. International Journal of Sports Physiology and Performance, 2019;14:902-10. https://doi.org/10.1123/ijspp.2018-0681

8. Tabor P, MastalerzA, Iwańska D \& Grabowska O. Asymmetry Indices in Female Runners as Predictors of Running Velocity. Polish Journal of Sport and Tourism. 2019;26(3): 3-8. https://doi.org/10.2478/pjst-2019-0013

9. Warmenhoven J, Smith R, Draper C, Harrison AJ, Bargary $\mathrm{N}$, Cobley S. Force coordination strategies in on-water single sculling: Are asymmetries related to better rowing performance? Scand J Med Sci Sports, 2018;28:1379-1388. https://doi.org/10.1111/sms.13031

10.Romanenko V, Veretelnikova N. Methods of evaluation of motor functional asymmetry of single combatants. Edinoborstva. 2020; 1(15): 67-77. https://doi.org/10.15391/ed.2020-1.07

11.Podrigalo L, Iermakov S, Romanenko V, Rovnaya O, Tropin Y, Goloha V, et al. Psychophysiological features of athletes practicing different styles of martial arts - the comparative analysis. International Journal of Applied Exercise Physiology, 2019;8:84-91. https://doi.org/10. 30472 /ijaep.v8i1.299

12.Nikolaenko NN, Mikheev MM, Afanas'ev SV. Changes of motor and sensory asymmetries in highly trained athletes. Journal of Evolutionary Biochemistry and Physiology. 2001;37(3): 273-279. https://oi.org/10.1023/A:1012623424655

13.Rovnaya O, Podrigalo L, Aghyppo O, Cieślicka M, Stankiewicz B. Study of Functional Potentials of Different Portsmanship Level Synchronous Swimming Sportswomen under Impact of Hypoxia. Research Journal of Pharmaceutical, Biological and Chemical Sciences. 2016; 7(4): 1210-1219.

14.Krstulovic S, Kuvacic G, Erceg M. Morphological, functional, and dynamical asymmetry in female judokas. Acta Kinesiologica. 2017;11(2):12-18.

15.Lewis V, Douglas JL, Edwards T, Dumbell L. A preliminary study investigating functional movement screen test scores in female collegiate age horse-riders. Comparative Exercise Physiology, 2019;15:105-12. https://doi.org/10.3920/CEP180036

16.Zhu J. Causes of Sports Injury of Badminton Players and Design of Forecasting Models. Investigacion Clinica. 2019; 60(1):87-97.

17. Marques VB, Medeiros TM, de Souza Stigger F, Nakamura FY, Baroni BM. The functional movement screen $\left(\mathrm{fms}^{\mathrm{tm}}\right)$ in elite young soccer players between 14 and 20 years: composite score, individual-test scores and asymmetries. Intl J Sports Phys Ther, 2017;12:977-85. https://doi.org/10.26603/ijspt20170977

18.de Oliveira RR, Chaves SF, Lima YL. There are no biomechanical differences between runners classified by the Functional Movement Screen. International Journal of Sports Physical Therapy. 2017;12(4): 625-633

19. Whittaker JL, Booysen N, de la Motte S, Dennett L, Lewis CL, Wilson D, et al. Predicting sport and occupational lower extremity injury risk through movement quality screening: a systematic review. Br J Sports Med, 2017;51:580-5. https://doi.org/10.1136/bjsports-2016-096760

20.Wieczorek M. Speed of learning complex motor skills and functional and dynamic asymmetry in 10-year old children. Physical Education \& Sport, 2001;45(1): 115-124.

21.Garcia-Garcia O, Cuba-Dorado A, Alvarez-Yates $\mathrm{T}$ et al. Clinical utility of tensiomyography for muscle function analysis in athletes. Open Access Journal of Sports Medicine. 2019;10: 49-69. https://doi.org/10.2147/OAJSM.S161485

22.Arboix-Alió J, Aguilera-Castells J, Rey-Abella F, Buscà B, Fort-Vanmeerhaeghe A. Asimetrías neuromusculares entre miembros inferiores en jugadores de hockey sobre patines. [Lower limb neuromuscular asymmetry in roller hockey players]. Rev Int Cienc Deporte, 2018;14:358-73. (In Spanish) https://doi.org/10.5232/ricyde2018.05406

23.Burmistrov AD, Chikurov AL, Khudik SS. 
Functional asymmetry of elite speed skaters. Tomsk State University Journal. 2018;434:143-148. https://doi.org/10.17223/15617793/434/19

24.Krzykała M, Leszczyński P, Grześkowiak M, Podgórski T, Woźniewicz-Dobrzyńska M, Konarska A, et al. Does field hockey increase morphofunctional asymmetry? A pilot study. Homo-JournalofComparative Human Biology, 2018;69:43-9. https://doi.org/10.1016/j.jchb.2018.03.003

25.Akinina MD, Sukhostayskaya KV, Kolpashnikova VS. Karate Classes as a Means of Motor and Psychomotor Training for Preschool Children. Tomsk State University Journal. 2019; 448: 187- 192. https://doi.org/10.17223/15617793/448/23

26.Hromcik A, Zvonar M, Balint G. Differences in Sensorimotor Skills between Badminton Players and Non-Athlete Adults. Brain-Broad Research in Artificial Intelligence and Neuroscience. 2019;10(2):47-54.
27.Podrigalo O, Borisova O, Podrigalo L, Iermakov S, Romanenko V, Bodrenkova I. The analysis of psychophysiological features of footbal players and water sports athletes. Phys Activ Rev. 2020; 8(1): 64-73. https://doi.org/10.16926/par.2020.08.08

28. Rodrigues PC, Vasconcelos O, Barreiros J, Barbosa R, Trifilio F. Functional asymmetry in a simple coincidence-anticipation task: Effects of handedness. European Journal of Sport Science, 2009;9:115-23. https://doi.org/10.1080/17461390802603903

29.Surina-Marysheva E, Erlikh V, Kantyukov S, Naumova K. Psychophysiological features in elite hockey players aged 15-16. Human Sport Medicine, 2019;19:36-41. https://doi.org/10.14529/hsm190105

\section{Information about the authors:}

Vyacheslav V. Romanenko; https://orcid.org/0000-0002-3878-0861; slavaromash@gmail.com; Kharkov State Academy of Physical Culture; Klochkovskaya str. 99, Kharkov, 61022, Ukraine.

Olha O. Podrihalo; http://orcid.org/0000-0003-1519-5632; rovnayaolga77@ukr.net; Kharkov State Academy of Physical Culture; Klochkovskaya str. 99, Kharkov, 61022, Ukraine.

Leonid V. Podrigalo; (Corresponding author); http://orcid.org/0000-0002-7893-524X; I.podrigalo@mail.ru; Kharkov State Academy of Physical Culture; Klochkovskaya str. 99, Kharkov, 61022, Ukraine.

Sergii S. lermakov; http://orcid.org/0000-0002-5039-4517; sportart@gmail.com; Department of Sport, Gdansk University of Physical Education and Sport; Kazimierza Górskiego 1, 80-336 Gdańsk, Poland.

Zhanna V. Sotnikova-Meleshkina; http://orcid.org/0000-0001-5534-8264; zhanna.univer@gmail.com; V. N. Karazin Kharkov National University; 4 Svobody Sq.,Kharkov, 61022, Ukraine

Oksana V. Bobrova; https://orcid.org/0000-0002-5953-1071; parryprof1@gmail.com;V. N. Karazin KharkovNational University; 4 Svobody Sq.,Kharkov, 61022, Ukraine.

Cite this article as:

Vyacheslav V. Romanenko, Olha O. Podrihalo, Leonid V. Podrigalo, Sergii S. lermakov, Zhanna V. Sotnikova-Meleshkina, Oksana V. Bobrova. The study of functional asymmetry in students and schoolchildren practicing martial arts. Physical Education of Students, 2020;24(3):154-161.

https://doi.org/10.15561/20755279.2020.0305

This is an Open Access article distributed under the terms of the Creative Commons Attribution License, which permits unrestricted use, distribution, and reproduction in any medium, provided the original work is properly cited http://creativecommons.org/licenses/by/4.0/deed.en

Received: 10.05.2020

Accepted: 15.06.2020; Published: 30.06 .2020 